\title{
The Formation of Close Binary Stars
}

\author{
Ian A. Bonnell \\ University of St Andrews, Physics and Astronomy, North Haugh, St \\ Andrews, KY16 9SS, UK
}

\begin{abstract}
I review the possible formation mechanisms of close binary stars. The formation of close binary systems is problematic in that there is no theory that does not encounter significant difficulties or unknowns. Fission does not appear to occur in stars. Capture is unlikely to form many close binary systems except possibly amongst massive stars. Fragmentation can form close binary systems but these need to accrete the majority of their eventual mass. Furthermore, there appears to be a limited window in initial conditions that may preclude forming sufficient systems in this way. Possible alternatives include the orbital migration of a binary due to its circumbinary disk and the disintegration of a nonhierarchical multiple system.
\end{abstract}

\section{Introduction}

Close binary stars (those with separations $\lesssim 1 \mathrm{AU}$ ) make up roughly $10 \%$ of all binary systems. They receive a larger proportion of astronomers' attention due to their potential for interaction and formation of exotic objects once they evolve off the main sequence (eg. Wijers, Davies \& Tout 1995). We know that close binaries exist in both main sequence and pre-main sequence populations (Mathieu 1994) and yet we still are far from certain how they form. Theories for binary star formation such as fragmentation generally have a hard time explaining the close systems whereas those that are designed to form close systems such as fission have been shown not to work. In this review I will cover some of the difficulties and advances in trying to develop a theory for the formation of close binary systems.

Firstly, it is worthwhile to review possible clues in the observed properties of close binary systems. The binary separation distribution shows the close binaries to represent a tail in a smooth, but very broad distribution (Duquennoy \& Mayor 1991). This can be interpreted as indicating one single mechanism, or at least the same mixture of mechanisms as wider binaries, but the distribution is so broad that it can be equally well represented as a uniform distribution in log separation. On the other hand, The mass ratio distribution does appear to be somewhat different (flatter) for close systems (Mazeh et al. 1992; see also chapter by J.-L. Halbwachs) than it is for wider systems (Duquennoy \& Mayor 1991) indicating that the formation mechanisms may indeed be different. 


\section{Overview of Formation Mechanisms}

We can divide up possible theories of close binary formation into two main branches. The first group involves forming the two components in situ, ie. at the separation we find them at. Such theories include fission and fragmentation. The second group involves the migration of the two components from larger separations to the closer separations. In the extreme case this is the capture of two unbound systems although a more probable scenario has the evolution of an already formed binary system (from say a fragmentation event) towards a more bound state by the extraction of orbital angular momentum. This could occur via the disintegration of a multiple system or via a circumbinary disk.

\section{Fission}

One of the earliest binary formation mechanisms, fission involves the splitting into two parts of a rapidly rotating protostar (Jeans 1919). It is ideally suited to forming close binaries as any resulting system will have a separation comparable to the initial object. Fission was hypothesised to occur after the initial formation phase has resulted in a quasi-equilibrium pressure-supported protostar. The idea is that as a protostar contracts, it will spin up due to angular momentum conservation. Analysis of the stability of rotating spheroids have shown that as the protostar's spin increases, it becomes unstable to non-axisymmetric perturbations and deforms into a bar-like object (cf. Tassoul 1978). This occurs once $\beta$, the ratio of rotational to the absolute value of the gravitational energies, is greater than 0.27 . As the bar-mode grows into the non-linear regime, the conjecture was that the bar would cleave into two pieces (eg. Roxburgh 1966; Bodenheimer \& Ostriker 1970).

Unfortunately, numerical simulations of the growth of the bar-mode into the non-linear regime have repeatedly shown that fission does not occur for compressible fluids such as stars (eg. Durisen et al. 1986). Both simulations starting from rotationally unstable initial conditions $(\beta>0.27$; Durisen et al. 1986) and those that follow the formation and spin-up of the protostar (Bonnell 1994; Bate 1998) have found similar results. Instead of fission, the bar develops spiral arms at each end which then extract angular momentum away from the central object by gravitational torques (Bonnell 1994). This transport of angular momentum outwards involves little of the protostar's mass but is able to remove the majority of the angular momentum. The end result is a circumstellar disk containing a small fraction of the total mass around a now stable protostar (Fig.1). Thus, fission has been abandoned as a binary formation mechanism.

\section{Capture}

Capture occurs when two stars pass close together in the presence of a dissipating medium which can remove the excess kinetic energy to leave the two stars bound. This medium can be a third star (eg. Binney \& Tremaine 1987), a circumstellar disk (Clarke \& Pringle 1991; Hall, Clarke \& Pringle 1996) or the stars themselves if the encounter is close enough to raise tides (Fabian et al. 1975). 


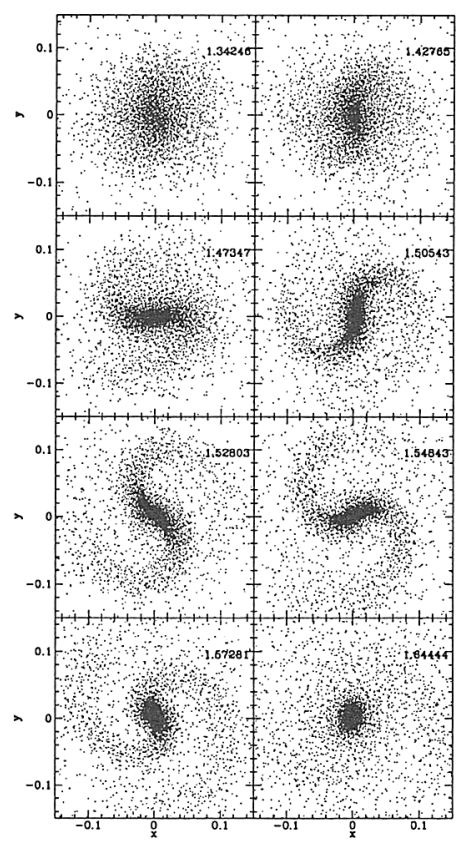

Figure 1. An SPH simulation showing the development of a rotational instability in a protostellar core formed at the end of a collapse phase (Bonnell 1994). The bar, and spiral arms, develop once $\beta>0.27$ and transport angular momentum outwards via gravitational torques leaving a rotationally stable protostar and surrounding disk.

In terms of forming close binaries, star-disk capture is unlikely to play a large role as the capture cross section is the disk size and thus would generally result in binaries of $\approx 100 \mathrm{AU}$. Three-body capture where the third star escapes with the excess kinetic energy requires high stellar densities and is not likely to be a major binary formation mechanism either (Binney \& Tremaine 1987).

The remaining capture mechanism, tidal capture, also requires high stellar density which is unlikely to be a general occurrence. Although this implies that tidal capture is unlikely to be responsible for the majority of close binaries, there is the possibility that tidal capture is responsible for the close binaries among massive systems (Bonnell et al. 1998). Massive star formation is difficult due to the radiation pressure on the infalling gas which can halt the accretion process (Yorke 1993). An alternative mechanism involves the ultra-dense core of a young stellar cluster which develops due to gas accretion. The young stars in this environment undergo direct collisions to build up the massive stars (Bonnell et al. 1998). In such a scenario, tidal capture occurs more frequently than collisions such that the resultant massive stars will commonly be in close binary systems. 

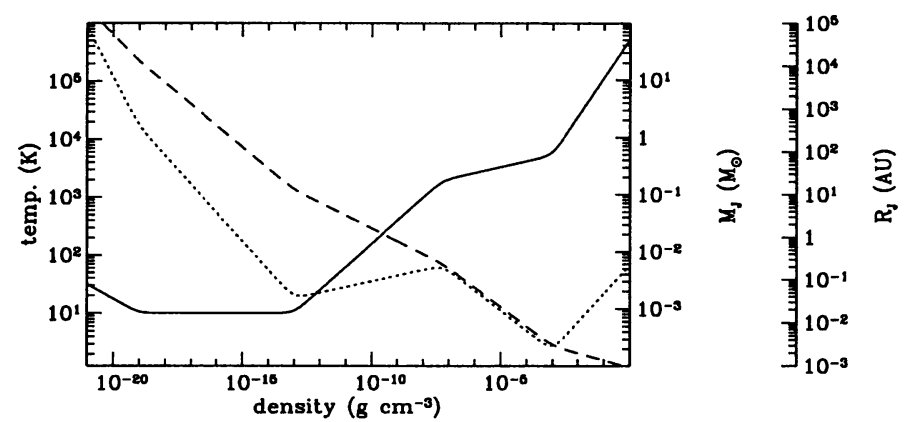

Figure 2. The temperature (solid line), Jeans mass (dotted line), and Jeans radius (dashed line) of interstellar gas as a function of density, showing the different phases the gas passes through during collapse (adapted from Tohline 1982).

\section{Fragmentation: Overview}

Fragmentation is probably the primary mechanism for the formation of most binary stars (Bonnell 1999; see chapter by P. Bodenheimer). It involves the separation into two or more parts, due to their self-gravity, of a dynamically evolving body. It is distinct from fission as it does not originate from a quasiequilibrium configuration. The problem with regards to forming close binary stars through fragmentation involves the dynamics of the collapse process and that at high densities the gas becomes optically thick.

In order for fragmentation to occur, the pre-fragment parts of the cloud must each contain a Jeans mass, the minimum mass to be gravitationally bound. In terms of a cloud of density $\rho$ and temperature $T$, the Jeans mass is

$$
M_{J}=\left(\frac{5 R_{g} T}{2 G \mu}\right)^{3 / 2}\left(\frac{4}{3} \pi \rho\right)^{-1 / 2}
$$

where $R_{g}$ is the gas constant, $G$ is the gravitational constant, and $\mu$ is the mean molecular weight. Thus, the cloud must contain at least two Jeans masses to fragment.

During the collapse, the density and the temperature (once the gas is optically thick) increase such that the Jeans mass changes too. The evolution of the temperature and of the Jeans mass during collapse is shown in figure 2 . The four phases of the collapse include an initial isothermal phase $(T \approx 10 \mathrm{~K})$ where the $M_{J}$ decreases throughout. Once the gas becomes optically thick to the IR radiation, a first protostellar core forms, and $T$ and $M_{J}$ both increase. A second collapse phase follows when the gas becomes hot enough to dissociate $\mathrm{H}_{2}$, the energy absorbed by this process destabilising the protostellar core until all the hydrogen is in atomic form. The temperature increases slightly during this phase whereas $M_{J}$ decreases. The last stage is the final protostellar core formed of atomic hydrogen where both the temperature and $M_{J}$ continue to increase.

In order to estimate when fragmentation will form close binaries systems, we can use the Jeans length, the minimum radius for an object to be gravitationally 
bound:

$$
R_{J}=\left(\frac{5 R_{g} T}{2 G \mu}\right)^{1 / 2}\left(\frac{4}{3} \pi \rho\right)^{-1 / 2} .
$$

This radius corresponds to half the minimum separation of the two fragments as otherwise they would overlap before fragmenting. The evolution of the Jeans radius during collapse (Fig. 2) shows how the Jeans radius decreases throughout the collapse. In order for the separation of the binary to be $\lesssim 1 \mathrm{AU}$, the fragmentation must occur at densities $\gtrsim 10^{-10} \mathrm{~g} \mathrm{~cm}^{-3}$, after the first protostellar core has formed.

Fragmentation can occur easiest during the collapse phases where $M_{J}$ are decreasing with increasing density. Generally, collapse during the isothermal phase (see chapter by P. Bodenheimer) requires some non-spherical configuration or perturbation that can grow duing the collapse. This is straightforward in this phase as there is no reason for the initial conditions to be spherical or equilibrium objects. Unfortunately, fragmentation during the second collapse phase is much more difficult due to the previous protostellar core which removes any non-spheroidal perturbations as it contracts quasistatically. Thus, any small perturbations that do exist in the $2^{\text {nd }}$ collapse phase are unlikely to lead to fragmentation (eg. Boss 1989).

\section{Rotationally Driven Disk Fragmentation}

An alternative to the direct fragmentation, that occurs in the isothermal collapse phase and that cannot occur during the $2^{\text {nd }}$ collapse phase, is disk fragmentation at the end of the collapse phase (Bonnell 1994). As a collapsing cloud spins up due to angular momentum conservation, the rotation can halt collapse and form a disk of material. All that is needed to fragment this disk is a way of generating structure and of removing the support due to the differential rotation.

As found in the case of fission, a rapidly rotating self-gravitating spheroid is unstable to the growth of a bar-mode which generates spiral arms and transports angular momentum outwards. This rotational instability occurs naturally at the end of a collapse phase when rotation is included, either in terms of a central partially pressure-supported protostar or the disk itself (Bonnell 1994). The main difference between this evolution and the fission instability is the presence of the surrounding disk of material and the continued infall. Both contribute mass to the system and the infall helps to drive this instability as it adds angular momentum to the system.

Once the central region of the collapsing cloud has $\beta>0.27$, it develops a bar-mode perturbation $(m=2)$. This bar grows and forms spiral arms at each end due to the differential rotation. The gravitational torques from the spiral arms operating on the central regions transports angular momentum outwards, which due to the differential rotation, increases the length of the spiral arms and hence the gravitational torques. Additionally, small differences between the transport rates for each arm grow and push the central object away from the centre of mass of the system (an $m=1$ mode). The combination of the spiral arms, the $m=1$ mode and the continued infall helps the spiral arms gather a Jeans mass together to form the secondary (Bonnell 1994). The angular momentum transport through the spiral arms reduces the rotational support and 


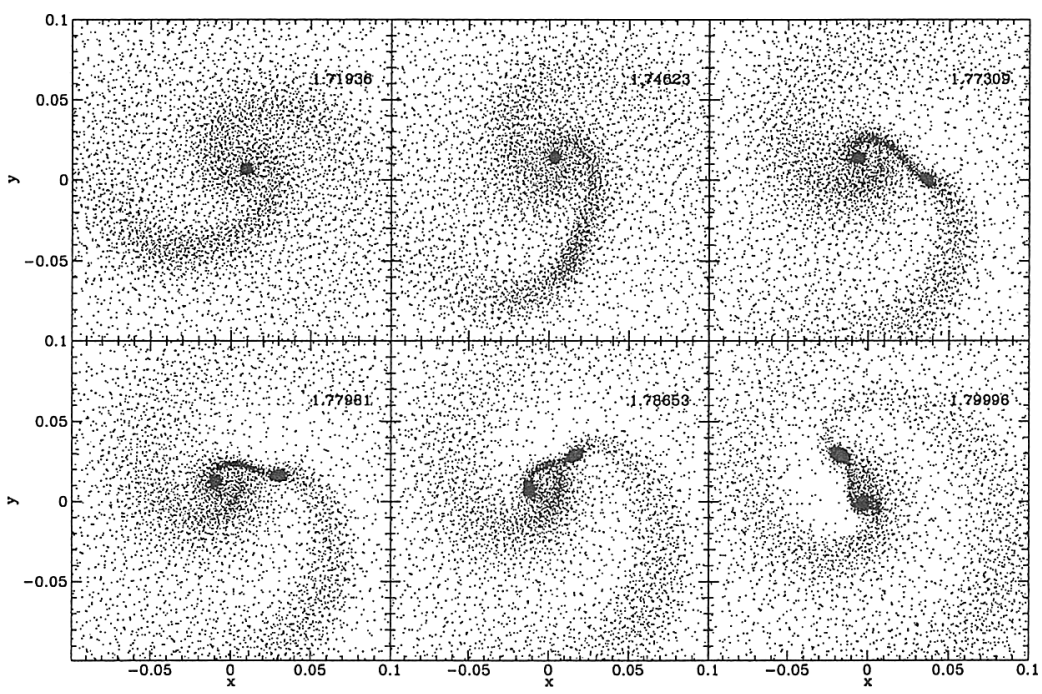

Figure 3. Protostellar disk fragmentation due to a rotational instability (Bonnell \& Bate 1994a).

the fragment can then collapse under its self-gravity. The continued infall is a crucial ingredient in this process and must add significant mass on a dynamical timescale which implies that this process occurs most easily just at the end of the collapse phase.

In order for this disk fragmentation to form close binaries, it has to occur at the end of the $2^{\text {nd }}$ collapse phase (Bonnell \& Bate 1994a). It follows the same process described above (see Fig. 3 ) except that, as it is at the end of the $2^{\text {nd }}$ collapse phase, less mass is involved and the resulting separation is $\approx 10 R_{\odot}$. The small mass of the binary $\left(m \approx 0.004 M_{\odot}\right)$ is due to the small amount of mass in the $2^{\text {nd }}$ collapse phase $\left(m \lesssim 0.01 M_{\odot}\right)$. This implies that any such system has to accrete $\approx 99 \%$ of its final mass and survive while doing so. Furthermore, the final properties of binaries formed in this way will depend almost entirely on the accretion process (Bate \& Bonnell 1997; Bate 2000; chapter by M. Bate).

While the future of the fragments is uncertain, they can engender further fragmentation in their relatively massive circumbinary disk which forms from the continued infall onto the system (Bonnell \& Bate 1994b). In this case, the binary plays the role of the $m=2$ bar-mode, driving spiral arms and an $m=1$ mode into the system (see Fig. 4). The interaction of the spiral arms and the infall then forms the additional fragments.

A potential difficulty with forming binaries through rotationally driven disk fragmentation at the end of the $2^{\text {nd }}$ collapse phase is that the initial conditions were chosen somewhat arbitrarily to represent the first protostellar core. A recent study has followed the collapse of a gas cloud from molecular cloud densities through all four phases of the collapse process to the formation of a near stellar density protostellar core (Bate 1998). This evolution found that for a relatively low initial value of rotational energy, $\beta=0.005$, the first protostellar core becomes rotationally unstable. The instability is not strong enough to fragment 


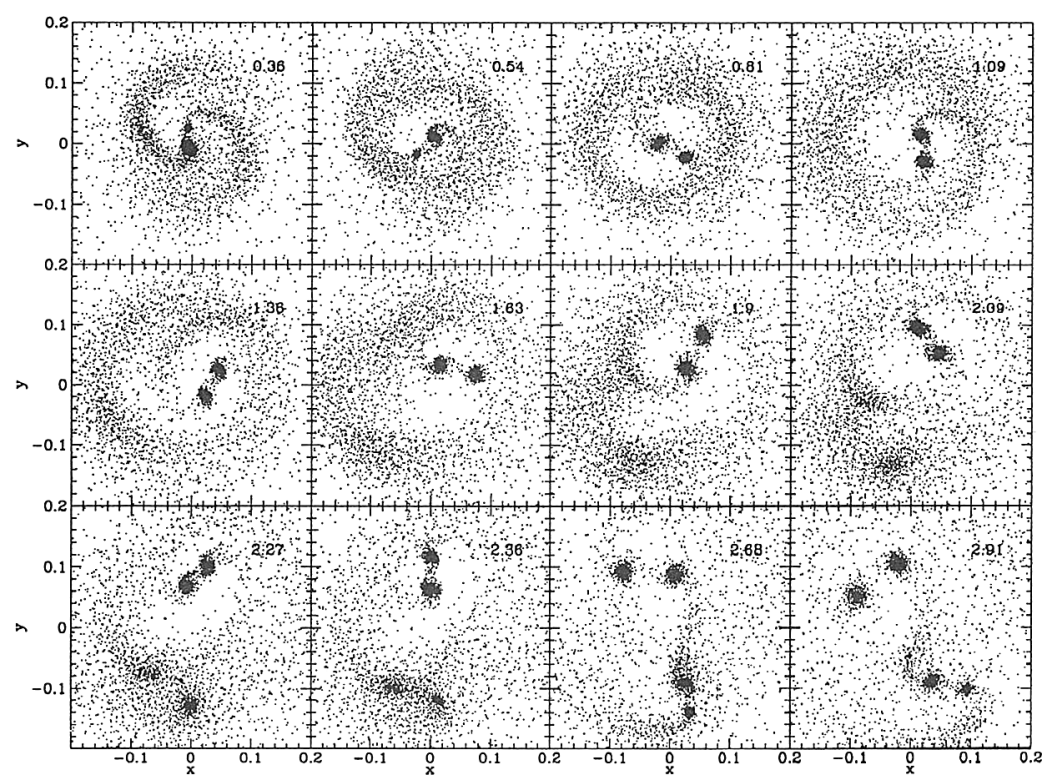

Figure 4. The fragmentation of a massive circumbinary disk due to the interaction with the central binary (Bonnell \& Bate 1994b).

but it does succeed in transporting large amount of angular momentum outwards from the central regions. So much so in fact that as the $2^{\text {nd }}$ collapse phase occurs, the angular momentum transport impedes any subsequent rotational instability (see Figure 5). In other words, the continuing angular momentum transport ensures that $\beta<0.27$ in the central regions and no close binary system is formed.

This implies that in order for the second core to go rotationally unstable, the first core must be rotationally stable. In order for this to happen, the initial conditions of the collapse must have very little rotational energy, $\beta \lesssim 10^{-4}$. This does pose a significant question mark over the whole process as to whether sufficient numbers of molecular clouds with such initial conditions exist in order to account for the frequency of close binary stars.

\section{Orbital Evolution}

An alternative to forming close binary stars in situ is that they formed with wider separations and due to some process were brought closer together. In such a scenario, the compenents can be formed in a more traditional fragmentation (eg. Bonnell et al. 1991; Boss 1993; Burkert \& Bodenheimer 1993) and can therefore be relatively massive straight away.

There are two possibilities as to how the system is made tighter. Firstly, if the system is formed with a circumbinary disk, then the gravitational torques of the binary on the disk transport angular momentum from the binary's orbit to the circumstellar disk (Pringle 1991; Artymowicz et al. 1991; Artymowicz 

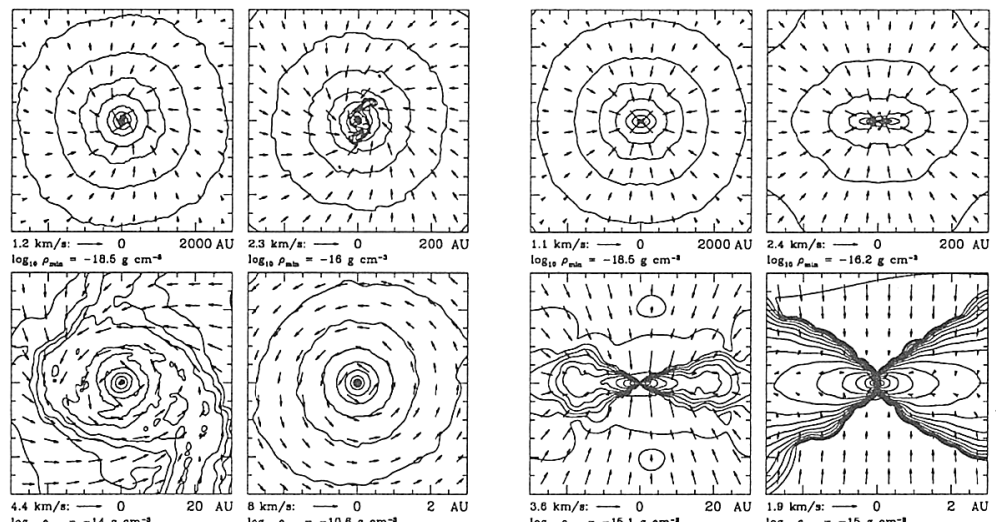

$4.4 \mathrm{~km} / \mathrm{s}:-20$
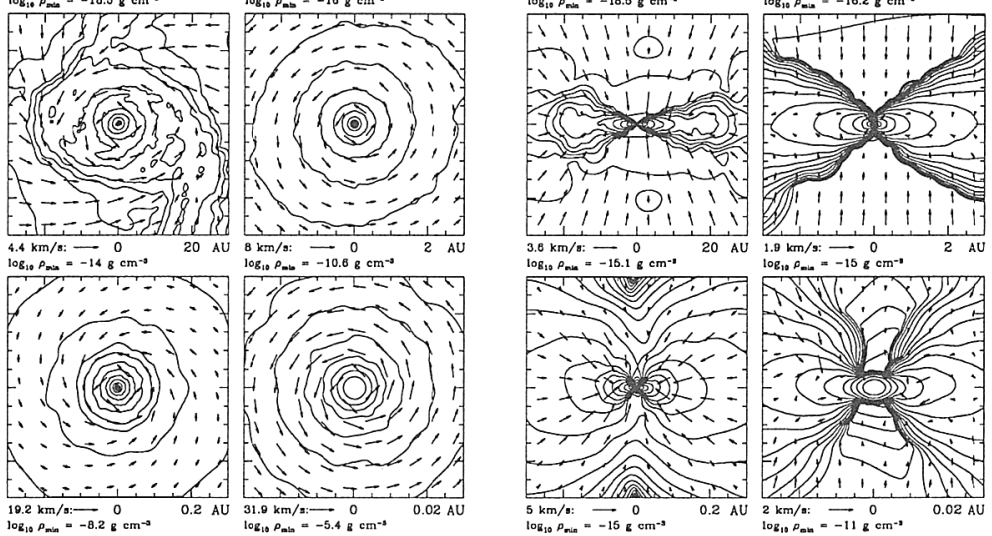

Figure 5. The final state of a collapse from interstellar to stellar densities. Density contours in the plane perpendicular (left panel) and parallel (right panel) to the rotation axis are shown (from Bate 1998).

\& Lubow 1994; see also chapter by P. Artymowicz). This process occurs on the disk's viscous timescale and can in principle extract an arbitrary amount of angular momentum from the binary. How such a process can explain the separation distribution within $10^{6}$ years, or why the systems should stop migrating at a given separation, and not merge, is presently unclear.

The second possibility is that the close systems form from the disintegration of a larger system containing multiple stars. Fragmentation simulations often produce multiple fragments and these systems are commonly non-hierarchical (see chapter by $\mathrm{P}$. Bodenheimer). These systems will disintegrate generally leaving the two most massive stars in a closer binary system (McDonald \& Clarke 1993; Sterzik \& Durisen 1998). The resultant separations are smaller than the initial separations (typically by a factor $\approx 0.1$ ) with a tail down to even smaller separations (see Fig.6); Sterzik \& Durisen 1999). It is still unclear if this scenario can sufficiently populate the close binary systems although further dissipation such as star-disc dissipation could play an important role (Clarke \& Pringle 1991b; McDonald \& Clarke 1995).

Support for the origin of the close binaries in higher order systems comes from recent work showing that spectroscopic binaries are commonly found in multiple systems (Tokovinin 1997). Furthermore, there is some evidence that the mass ratio distribution is consistent with what is found from the dynamical interactions (Valtonen 1998). 


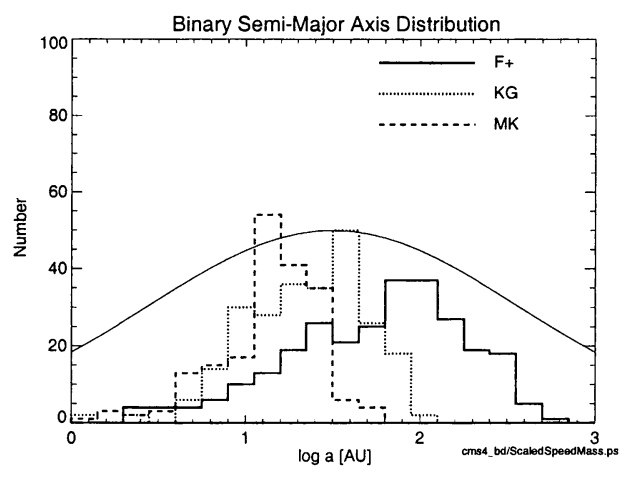

Figure 6. The distribution of separations that result from the disintegration of non-hierarchical systems is shown (from Durisen \& Sterzik 1999). Note that there is a tail of systems to smaller separations, although the numbers are probably insufficient to explain the close binary frequency.

\section{Summary}

Although significant progress has been made in our understanding of how binary stars in general form, there is still a large question mark as to how close binaries (separations $\lesssim 1 \mathrm{AU}$ ) form. Of the in situ theories, fission has been discounted as a formation mechanism as it does not occur. Fragmentation, at the end of the second collapse phase due to a rotational instability, can form close binary systems but with little mass. These fragments need to accrete the majority of their final stellar mass (see chapter by M. Bate). A further problem for the fragmentation process is that there appears to be a limited window in parameter space for this to occur which may prove problematic.

Alternatives to the in situ formation mechanisms involve capture and the orbital evolution of already formed binaries. Capture is unlikely to play a large role in forming most close binaries although it could be important if massive stars form through a collisional build-up in the dense core of young clusters. More promising is the orbital migration of binary systems due to their interaction with a circumbinary disk although it is unclear whether this process will yield the desired separations. Lastly, the disintegration of a non-hierarchical multiple system could also be responsible for close binaries, although additional orbital dissipation may be required.

In summary, it is clear that forming close binary systems is complex and that it probably involves several different physical processes that need to be included in a general model.

\section{References}

Artymowicz, P., Clarke, C. J., Lubow, S. H., Pringle, J. E. 1991, ApJ, 370, L35 Artymowicz, P., Lubow, S. H. 1994, ApJ, 421, 651

Bate, M. R. 1998, ApJ, 508, L95 
Bate, M. R. 2000, MNRAS, 314, 33

Bate, M. R., Bonnell, I. A. 1997, MNRAS, 285, 33

Bonnell, I. A. 1994, MNRAS, 269, 837

Bonnell, I. A. 1999, in The Origin of Stars and Planetary Systems, ed. C. Lada \& N. Kylafis, (Dordrecht), 479

Bonnell, I. A., Bate, M. R. 1994a, MNRAS, 269, L45

Bonnell, I. A., Bate, M. R. 1994b, MNRAS, 271, 999

Bonnell, I. A., Bate, M. R., Zinnecker, H. 1998, MNRAS, 298, 93

Bonnell, I. A., Martel, H., Bastien, P., Arcoragi, J.-P., Benz, W. 1991, ApJ, 377, 553

Boss, A. P. 1989, ApJ, 346, 336

Boss, A. P. 1993, ApJ, 410, 157

Burkert, A., Bodenheimer, P. 1993, MNRAS, 264, 798

Clarke, C. J., Pringle, J. E. 1991a, MNRAS, 249, 584

Clarke, C. J., Pringle, J. E. 1991b, MNRAS, 249, 588

Durisen, R. H., Gingold, R. A., Tohline, J. E., Boss, A. P. 1986, ApJ, 305, 281

Fabian, A. C., Pringle, J. E., Rees, M. J. 1975, MNRAS, 172, 15

Hale, A. 1994, AJ, 107, 306

Hall, S. M., Clarke, C. J., Pringle, J. E. 1996, MNRAS, 278, 303

Jeans, J. 1919, Problems of Cosmogony and Stellar Dynamics, (Cambridge U.P.)

Larson, R. B. 1969, MNRAS, 145, 271

Mathieu, R. D. 1994, ARA\&A, 32, 465

Mazeh, T., Goldberg, D., Duquennoy, A., Mayor, M. 1992, ApJ, 401, 265

McDonald, J. M., Clarke, C. J. 1993, MNRAS, 262, 800

McDonald, J. M., Clarke, C. J. 1995, MNRAS, 275, 671

Pringle, J. E. 1991, MNRAS, 248, 759

Roxburgh, I. 1966, ApJ, 143, 111

Sterzik, M., Durisen, R. 1998, A\&A, 339, 95

Sterzik, M., Durisen, R. 1999, in Star Formation 1999, ed. T. Nakamoto, Nobeyama Radio Observatory, 387

Tassoul, J. L. 1978, Theory of Rotating Stars, (Princeton U.P.)

Tohline, J. E. 1982, Fund. of Cos. Phys., 8, 1

Wijers, R., Davies, M. B., Tout, C. A. 1995, Evolutionary Processes in Binary Stars, (Dordrecht)

Yorke, H. W. 1993, in Massive Stars: Their Lives in the interstellar Medium, ed. J. Cassinelli, E. Churchwell, ASP Conf. Ser., 35, 45 